\title{
Pregnancy while using a levonorgestrel-releasing intrauterine system 52 mg: a case report
}

\author{
Elvin Piriyev*, Thomas Römer
}

Department of Obstetrics and Gynaecology, Academic Hospital Cologne Weyertal, Cologne, Germany

Received: 25 August 2020

Accepted: 01 October 2020

\section{*Correspondence:}

Dr. Elvin Piriyev,

E-mail: piriyev.elvins@gmail.com

Copyright: (C) the author(s), publisher and licensee Medip Academy. This is an open-access article distributed under the terms of the Creative Commons Attribution Non-Commercial License, which permits unrestricted non-commercial use, distribution, and reproduction in any medium, provided the original work is properly cited.

\begin{abstract}
Intrauterine contraception mostly in the form of copper intrauterine device (IUD) or hormone-releasing system, is a widely used method with a high level of contraceptive security. Despite high contraceptive efficiency, pregnancy can still occur. Pregnancy with a levonorgestrel-releasing intrauterine system (LNG-IUS) in situ is very rare. Intracavitary pregnancy with an IUD can lead to a higher risk of infection and preterm birth. Present the case of a 28-year old woman who was admitted to our hospital due to pregnancy with an LNG-IUS $52 \mathrm{mg}$ in the $9^{\text {th }}$ gestational weeks (8 weeks 3 days). Sonography showed an intact pregnancy corresponding to the gestational week. The IUS was located in the lower part of the cavity at a considerable distance from the amniotic cavity. After extensive information on the benefits and risks, sonography-guided saline infusion hysteroscopy was carried out. The intact pregnancy and the IUS could be shown hysteroscopically. The removal of the IUS was carried out with a grasping forceps. There was no complication. After an inconspicuous course of pregnancy, the patient spontaneously gave birth in 37 gestational weeks 1 day.
\end{abstract}

Keywords: Risk pregnancy, LNG-IUS, Copper IUD, Pregnancy, Hysteroscopy

\section{INTRODUCTION}

Intrauterine contraception, usually in the form of copper IUD or LNG-IUS, is a widely used method with high contraceptive security. Complications include ascending genital infection, bleeding disorders, the occurrence of extrauterine pregnancy, as well as perforations and dislocations. Despite the high contraceptive efficiency, pregnancy can still occur. The failure rate of the intrauterine device as a contraceptive method is around 12 pregnancies per 100 women year (pearl index: copper spiral 0.9-3.0 failure/10 years, LNG-IUS $52 \mathrm{mg} 0.16$ failure/10 years, Gynefix 0-2.5 failures/10 years). ${ }^{12}$ An essential factor in developing a pregnancy under a nonhormonal IUD is luxation of the device. The fact that IUD dislocations are most common in the first year shows the need for more frequent controls during this period. ${ }^{9}$ Pregnancy with an LNG-IUS in situ is very rare. Ectopic pregnancies account for $53 \%$ of all pregnancies with the use of LNG-IUS. ${ }^{2}$ The rate of ectopic pregnancies with LNG-IUS is not increased compared to women who do not use IUS. In fact, ectopic pregnancies are less common when using contraception with any intrauterine contraceptive device than without contraception. ${ }^{1,3,8}$ Intracavitary pregnancy with an IUD can lead to a higher risk of infection and preterm birth. ${ }^{4,5,7}$ The studies show that the risk of intrauterine infection is increased especially if the pregnancy develops within the first three to four months of IUD insertion. ${ }^{5}$ Women who become pregnant with an intrauterine device have an increased risk of undesirable obstetric consequences. The risk of pregnancies with retained IUD is higher compared to the early removal of IUD.

\section{CASE REPORT}

Present the case of a 28-year-old GII/PI woman who was admitted to us due to pregnancy with LNG-IUS $52 \mathrm{mg}$ in 
the $9^{\text {th }}$ gestational weeks. Sonography showed an intact pregnancy corresponding to the gestational week. The IUS was located in the lower part of the cavity at a considerable distance from the amniotic cavity.

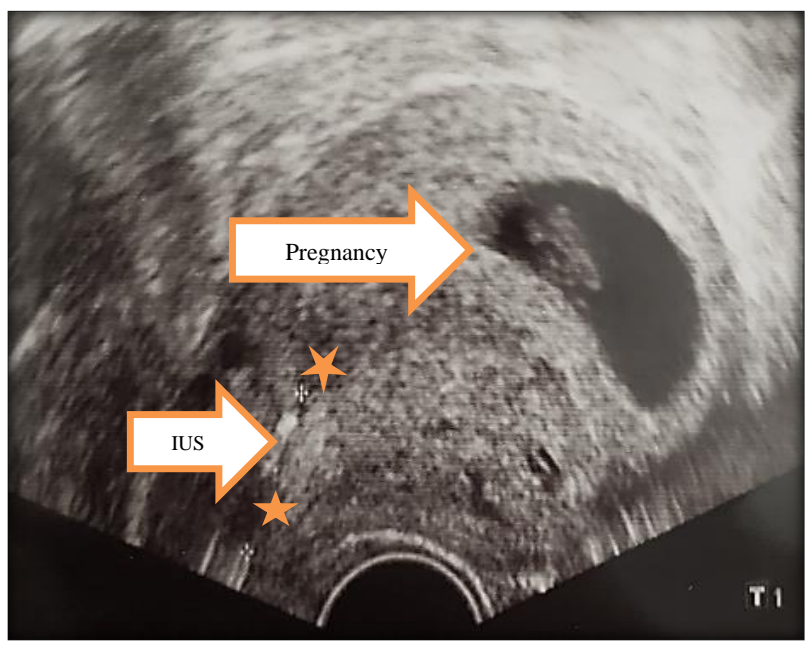

Figure 1: Pregnancy with IUS.

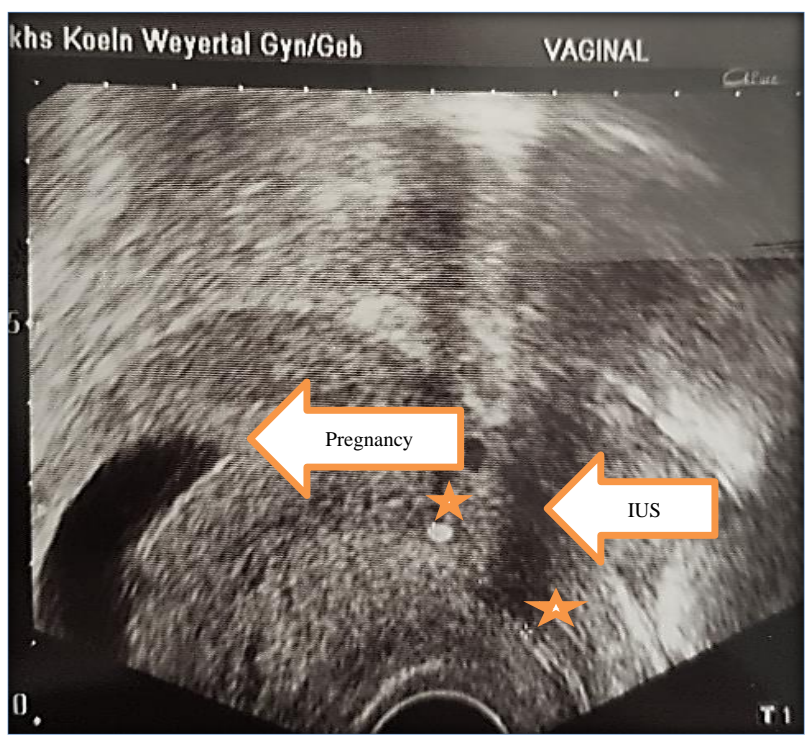

Figure 2: Pregnancy with IUS.

The patient wanted to remain pregnant, so the following options were discussed: Removal of the IUS under hysteroscopic control and pregnancy with IUS. She was informed that a pregnancy with an IUS is associated with preterm deliveries and an increased risk of infection. After receiving comprehensive information of both options, the patient decided to remove the IUS in the $10^{\text {th }}$ gestational week (9 weeks 3 days). The procedure was performed in spinal anesthesia. After grasping the cervix with two tenaculum, a hysteroscopy was performed under sonographic control. Pregnancy was displayed under low pressure with saline solution. Beside it, in the lower part of the cavity, the IUS was separate and could be easily identified with threads. The IUS was carefully extracted with a grasping forceps. Subsequently, a sonographic control was carried out which showed unaffected amniotic cavity and positive heart activity. The patient remained hospitalized for one night. She did not have postoperative relevant bleeding. Four hours postoperatively and on the following day, a sonographic check was carried out, which continued to show an intact pregnancy. The patient was discharged with the recommendation of further pregnancy checks according to the maternity guidelines. Further controls were carried out in an outpatient practice and were inconspicuous. The patient gave birth spontaneously in 37 weeks 1 day after an unremarkable course of pregnancy. The healthy child weighed $2900 \mathrm{gm}$.

\section{DISCUSSION}

The use of IUD is an alternative to hormonal oral contraception. Pregnancy with an IUD use is a rare event. ${ }^{6}$ Delivering a baby with an IUD is very rare and is associated with an increased risk of preterm birth and infection. Several authors reported complications in pregnancies with an IUD. Brahmi et al conducted an analysis and showed that women with a retained IUD had the greatest risk of adverse pregnancy outcomes, including spontaneous abortion, preterm birth, septic abortion and chorioamnionitis. ${ }^{4}$ The removal of copper IUD reduced the risk. Case series with LNG-IUS showed that when retained in situ, 8 out of 10 pregnancies ended with a spontaneous abortion. ${ }^{4}$ They concluded that the pregnancies, which were complicated by a retained IUD in situ, had a higher risk of adverse pregnancy outcomes. An early removal of the IUD seemed to improve the results, but did not completely eliminate the risks. ${ }^{4}$ Chaim et al also recommended the removal of the IUD in the early weeks of pregnancy. They compared 16 women who became pregnant while using an IUD with a control group of 48 women during a normal pregnancy. The use of IUD before removal during pregnancy was between 3 and 39 months (median value 15.5 months). The rate of preterm birth was significantly higher in the study group than in the control group (18.7 vs $2 \%) .^{5}$

Sanders et al performed a saline infusion hysteroscopy with and/or without simultaneous ultrasound guidance with removal of the IUD in 26 pregnant women. The average gestational age at the time of the intervention was 11 weeks. In 22 out of 26 cases (84.6\%) successful IUD removal has been carried out. There were 23 live births, including 20 full-term and three preterm births. The average gestational age in childbirth was 38 weeks 4 days. After the intervention, there was one miscarriage and one elective abortion. There were no complications directly related to the procedure. ${ }^{11}$ They concluded that saline infusion hysteroscopy is a safe and effective method of removing IUD in early pregnancy. ${ }^{11}$

Ganer et al carried out a retrospective study comparing the pregnancy outcome in women with an IUD $(n=98)$, women after removal of IUD in the early weeks of pregnancy $(n=194)$ and pregnant women without an IUD 
$(n=141191)$. The rate of preterm birth was $18.4 \%$ in the group with an IUD, $14.4 \%$ in the group with a removed IUD and $7.3 \%$ in the group of patients who never had an IUD during their pregnancy. Chorioamnionitis was found in the group with an IUD at $7.1 \%$, in the group with a removed IUD at $4.1 \%$ and in the group without IUD in $0.7 \%$ of the women. ${ }^{7}$ Kim et al came to the same conclusion. They examined 196 pregnancies with IUD and discovered that pregnancies with an IUD had a higher rate of a late miscarriage, preterm birth, vaginal bleeding, clinical chorioamnionitis and placenta abruption than those without IUD. Similarly, histological examination of the placenta revealed a higher rate of histological chorioamnionitis in patients with an IUD compared to patients without IUD (54.2\% vs. $14.7 \%)$. In patients with amniocentesis, the prevalence of amniotic cavity infection was higher in pregnant women with an IUD than in patients without an IUD (45.9\% vs. $8.8 \%)$. Intra-amniotic candida infection was also more common in pregnancies with an IUD than in those without an IUD $(31.1$ vs $6.3 \%){ }^{10}$

Childbirth complications can occur with IUD use. Dominik et al described a case of pregnancy with a retained intrauterine system. The IUD was found partially in the child's left tonsil and partially in the child's hypopharynx after birth. The IUD could be removed. The subsequent examination of the child's throat showed a clear impression of the left tonsil. ${ }^{6}$

Backman et al came to following conclusion: If pregnancy occurs while using LNG-IUS, there is an increased risk of ectopic pregnancy. They analyzed 17360 addressed users who answered the questionnaire. A total of 132 pregnancies were reported. Medical records were checked on 108 of the pregnant women. In 64 pregnancies, a conception with the LNG-IUS in situ occurred. 33 pregnancies were ectopic. ${ }^{2}$

\section{CONCLUSION}

Based on the data, the removal of an IUD is recommended in the early weeks of pregnancy if the IUD is in a favorable location. While an early removal of an IUD decreases the risk of premature birth and an infection, the risks cannot be completely eliminated. We recommend the removal of an IUD in the 9-11 ${ }^{\text {th }}$ gestational week. In the first weeks of pregnancy (earlier than $9^{\text {th }}$ week) this could lead to a spontaneous abortion. In addition, the distance between the pregnancy and the IUD cannot be clearly differentiated within the first weeks of pregnancy. Upon removal of the IUD after 11 gestational weeks, risks for iatrogenic causing miscarriages increases. Besides general surgical risks, the patient must be explicitly informed about the risk of miscarriage.

Funding: No funding sources

Conflict of interest: None declared

Ethical approval: Not required

\section{REFERENCES}

1. Andersson K, Odlind V, Rybo G. Levonorgestrelreleasing and copper-releasing (Nova T) IUDs during five years of use: a randomized comparative trial. Contraception. 1994;49(1):56-72.

2. Backman T, Rauramo I, Huhtala S, Koskenvuo M. Pregnancy during the use of levonorgestrel intrauterine system. Am J Obstet Gynecol. 2004;190(1):50-4.

3. Benagiano G, Gabelnick H, Farris M. Contraceptive devices: intravaginal and intrauterine delivery systems. Expert Rev Med Devices. 2008;5(5):63954.

4. Brahmi D, Steenland MW, Renner RM, Gaffield ME, Curtis KM. Pregnancy outcomes with an IUD in Situ: a systematic review. Contraception. 2012;85(2):131-9.

5. Chaim W, Mazor M. Pregnancy with an intrauterine device in situ and preterm delivery. Arch Gynecol Obstet. 1992;252(1):21-4.

6. Dominik B, Schneider J. Pregnancy and IUS. Geburtshilfe Frauenheilkd. 1997;57(12):687-8.

7. Ganer H, Levy A, Ohel I, Sheiner E. Pregnancy outcome in women with an intrauterine contraceptive device. Am J Obstet Gynecol. 2009;201(4):381:e1-5.

8. Heinemann K, Reed S, Moehner S, Minh TD. Comparative contraceptive effectiveness of levonorgestrel-releasing and copper intrauterine devices. Eu Active Surveillance Study IUD. Contraception. 2015;91(4):280-3.

9. Inal MM, Ertopçu K, Ozelmas I. The evaluation of 318 intrauterine pregnancy cases with an intrauterine device. Eur J Contracept Reprod Health Care. 2005;10(4):266-71.

10. Kim SK, Romero R, Kusanovic JP, Erez O, Vaisbuch E, Mazaki-Tovi S et al. The prognosis of pregnancy conceived despite the presence of an intrauterine device (IUD). J Perinat Med. 2010;38(1):45-53.

11. Sanders AP, Fluker MR, Sanders BH. Saline Hysteroscopy for Removal of Retained Intrauterine Contraceptive Devices in Early Pregnancy. J Obstet Gynaecol Can. 2016;38(12):1114-9.

12. Wallwiener M, Friedrich $O$, Schäfer K, Thum J, Eichbaum M, Rom J. Intrauterinpessare. Ultrasound in gynecology and obstetrics. Thieme, Stuttgart. 2013;3.

Cite this article as: Piriyev E, Römer T. Pregnancy while using a levonorgestrel-releasing intrauterine system 52 mg: a case report. Int J Reprod Contracept Obstet Gynecol 2020;9:4692-4. 